\title{
O papel do enfermeiro na educação sexual dos adolescentes
}

\section{The role of nurses in the sexual education of adolescents \\ El papel de las enfermeras en la educación sexual de los adolescentes}

Silvia Maria da Silva Sant'ana Rodrigues ORCID: https://orcid.org/0000-0003-2421-8701 Centro Universitário Uninassau, Brasil

E-mail: profenf.silviasantana@gmail.com

Thaynara de Almeida Melo ORCID: https://orcid.org/0000-0001-8430-5458 Centro Universitário Uninassau, Brasil E-mail: thayallmeida@hotmail.com

Camila Brunelle Pereira Santos ORCID: https://orcid.org/0000-0001-8406-6178 Centro Universitário Uninassau, Brasil E-mail: camilabpstos@gmail.com

Weber de Santana Teles ORCID: https://orcid.org/0000-0003-1770-8278 Centro de Hemoterapia de Sergipe, Brasil E-mail: arteecura@hotmail.com

Max Cruz da Silva

ORCID: https://orcid.org/0000-0002-6944-5986 Faculdade Pio Décimo, Brasil E-mail: maxlfi@hotmail.com

Ruth Cristini Torres

ORCID: https://orcid.org/0000-0002-8664-192X Instituto de Hematologia e Hemoterapia de Sergipe, Brasil E-mail: ruthcristini@gmail.com

Marcel Vinícius Cunha Azevedo ORCID: https://orcid.org/0000-0002-5312-3333 Universidade Tiradentes, Brasil

E-mail: marcelvinicius49@gmail.com

Taíssa Alice Soledade Calasans ORCID: https://orcid.org/0000-0003-0460-4437 Universidade Tiradentes, Brasil E-mail: taissa.asc@gmail.com

Maria Hozana Santos Silva ORCID: https://orcid.org/0000-0001-5742-5366

Faculdade Ages de Medicina, Brasil E-mail: hosana_p@hotmail.com

Aline Barreto Hora

ORCID: https://orcid.org/0000-0002-3930-6475 Centro Universitário Estácio de Sergipe, Brasil E-mail: aline.barretoh@hotmail.com

Ana Fátima Souza Melo de Andrade ORCID: https://orcid.org/0000-0002-7024-6175

Centro Universitário Estácio de Sergipe, Brasil E-mail: anafatimamelo@hotmail.com

Ângela Maria Melo Sá Barros ORCID: https://orcid.org/0000-0003-4087-3247 Universidade Federal do Rio de Janeiro, Brasil E-mail: angelsamelo@hotmail.com

Paulo Celso Curvelo Santos Junior ORCID: https://orcid.org/0000-0001-5834-6782 Universidade Tiradentes, Brasil E-mail: paulo.curvelo.jr@gmail.com

\section{Resumo}

O presente estudo tem como objetivo verificar o papel do enfermeiro no cuidado a saúde do adolescente, descrevendo ações prestadas para auxiliá-los em casos de gestação precoce, e enfatizar a importância de utilizar métodos contraceptivos para prevenção de infecções sexualmente transmissíveis. Trata-se de uma revisão integrativa de literatura 
com artigos já publicados, foram selecionados artigos de acordo com os critérios de inclusão. Após leitura e coleta de dados da temática, os resultados mostraram que adolescentes têm iniciado a relação sexual precocemente, apesar de terem conhecimento sobre o assunto abordado, existe uma porcentagem desse grupo vulnerável as infecções sexualmente transmissíveis, devido à diminuição e/ou a falta da orientação a saúde sexual. O apoio familiar e o acolhimento dos profissionais de saúde em casos de gravidez precoce é fundamental, para acompanhar a saúde da adolescente e do bebê durante as consultas, afim de prevenir os riscos na gestação que levem a complicações futuras. A troca de informações entre adolescentes e o enfermeiro é imprescindível, pois aprimora o conhecimento dos jovens e os aproxima dos profissionais de saúde, através das ações educativas. É necessário entender a realidade para que tenha intervenções objetivas, com resultado positivo e participação dos jovens em todo dinamismo, assim existindo diálogo e momento de reflexões das experiências vividas. Entende-se que para redução desses agravos é necessário que o enfermeiro elabore técnicas de ações educativas para despertar o interesse dos adolescentes e manter uma troca de saberes entre os grupos formados, assim proporcionando qualidade de vida e educação em saúde.

Palavras-chave: Saúde do adolescente; Educação em saúde; Anticoncepção; Infecções sexualmente transmissíveis.

\begin{abstract}
This study aims to verify the role of nurses in adolescent health care, describing actions taken to help them in cases of early pregnancy, and emphasize the importance of using contraceptive methods to prevent sexually transmitted infections. It is an integrative literature review with articles already published, articles were selected according to the inclusion criteria. After reading and collecting data on the topic, the results showed that adolescents have started sexual intercourse early, despite having knowledge about the subject, there is a percentage of this group vulnerable to sexually transmitted infections, due to the decrease and/or lack of sexual health orientation. Family support and the welcoming of health professionals in cases of early pregnancy is essential to monitor the health of the adolescent and the baby during consultations, in order to prevent risks during pregnancy that lead to future complications. The exchange of information between adolescents and nurses is essential, as it improves the knowledge of young people and brings them closer to health professionals, through educational activities. It is necessary to understand the reality so that there are objective interventions, with positive results and the participation of young people in all dynamism, thus existing dialogue and a moment of reflection on the lived experiences. It is understood that, in order to reduce these problems, it is necessary for nurses to develop techniques of educational actions to awaken the interest of adolescents and maintain an exchange of knowledge between the groups formed, thus providing quality of life and health education.
\end{abstract}

Keywords: Adolescent health; Health education; Contraception; Sexually transmitted infections.

\title{
Resumen
}

Este estudio tiene como objetivo verificar el papel de las enfermeras en la atención de la salud de los adolescentes, describiendo las acciones tomadas para ayudarlas en los casos de embarazo precoz y enfatizar la importancia del uso de métodos anticonceptivos para prevenir las infecciones de transmisión sexual. Es una revisión de la literatura integradora con artículos ya publicados, los artículos fueron seleccionados de acuerdo con los criterios de inclusión. Luego de leer y recolectar datos sobre el tema, los resultados mostraron que los adolescentes han iniciado relaciones sexuales temprano, a pesar de tener conocimientos sobre el tema, existe un porcentaje de este grupo vulnerable a las infecciones de transmisión sexual, debido a la disminución y / o falta de relaciones sexuales. Orientación a la salud. El apoyo familiar y la acogida de los profesionales de la salud en los casos de embarazo precoz es fundamental para vigilar la salud de la adolescente y del bebé durante las consultas, con el fin de prevenir riesgos durante el embarazo que conlleven futuras complicaciones. El intercambio de información entre adolescentes y enfermeras es fundamental, ya que mejora el conocimiento de los jóvenes y los acerca a los profesionales de la salud, a través de actividades educativas. Es necesario comprender la realidad para que haya intervenciones objetivas, con resultados positivos y la participación de los jóvenes con todo dinamismo, existiendo así el diálogo y un momento de reflexión sobre las experiencias vividas. Se entiende que, para reducir estos problemas, es necesario que los enfermeros desarrollen técnicas de acciones educativas para despertar el interés de los adolescentes y mantener un intercambio de conocimientos entre los grupos formados, brindando así calidad de vida y educación para la salud.

Palabras clave: Salud de los adolescentes; Educación para la salud; Anticoncepción; Infecciones de transmisión sexual.

\section{Introdução}

Os adolescentes fazem parte de um grupo bastante vulneral e influenciável, e por isso necessitam de cuidados e atenção especiais. Com o crescente número de casos de Infecções Sexualmente Transmissíveis (IST) e gravidez não planejada entre os jovens, nos levou a refletir sobre a sexualidade dos adolescentes e a importância em reduzir problemas futuros, no que diz respeito à vida pessoal e social desses jovens (Almeida et al., 2017).

A antecipação da primeira relação sexual dos adolescentes está relacionada a modificações biológicas. O desenvolvimento psicológico se dá pela busca da identidade sexual que contribui para que os adolescentes experimentem novas 
sensações por meios de práticas sexuais. Em relação ao ponto de vista social, a vida sexual dos adolescentes está relacionada à vida econômica, religião e a escolaridade de cada indivíduo (Brêtas et al., 2011).

Sabe-se que as experiências sexuais que ocorrem nessa fase da vida são manifestadas através de práticas sexuais muitas vezes desprotegidas, devido à falta de informação. Observa-se que a exposição de jovens a múltiplos parceiros sexuais, tem levedo a problemas de saúde e gerado agravos, aliado ao uso incorreto ou ao não uso de contraceptivos que ocorrem desde o início da vida sexual (Camargo \& Ferrari, 2009).

É importante ressaltar que a escola juntamente com o os profissionais da saúde devem manter um contato, através do Programa Saúde nas Escolas (PSE), e desenvolver encontros para fazer palestras, conversas, orientações com troca de informações com aos adolescentes com o intuito de prevenir dos riscos à saúde.

Observando que a escola é o local com maior quantidade de adolescente em fase de mudanças hormonais, sociais e psicológicas. Dessa forma esse lugar foi escolhido como base para ser feita uma revisão integrativa para obter dados suficientes para que seja elaborado um plano de ação que ajudem esses adolescentes a compreender melhor sobre as infecções sexualmente transmissíveis, o uso correto do preservativo e prevenir uma gravidez indesejada (Pariz, Mengarda \& Frizzo, 2012).

Sabe-se que o enfermeiro também tem o papel de educador e com isso evidenciar o papel da educação sexual no ambiente escolar, visto que a escola é um local para o aprendizado. Com isso ajudar os jovens com orientações e assim contribuir para o esclarecimento dos fatores que predispõe aos adolescentes a iniciar-se sexualmente, as implicações da iniciação sexual precoce na sua vida reprodutiva, bem como as questões que envolvem a sua primeira experiência sexual como, o que eles entendem sobre Infecções Sexualmente Transmissíveis (IST), gravidez na adolescência e o não uso de métodos contraceptivos (Fonseca, Gomes \& Teixeira 2010).

A saúde sexual dos adolescentes tem alguns desafios, observa-se que a uma grande instabilidade em alguns grupos de jovens, tornando mais vulneráveis, uma elevada carga de doenças relacionadas, a gravidez não planejada, as infecções sexualmente transmissíveis. Esse fato tem ocorrido devido à falta de informação, fatores culturais e socias, violência e abuso sexual. Estudos mostram que em 2015, 102.072 estudantes que estavam regularmente matriculados na nona série do ensino fundamental, 27,5\% desses jovens relatam já terem iniciado a vida sexual. O uso de preservativo é de 66,2\%, e 19,5\% que não usam nenhum contraceptivo, 36,1\% dos que usam apenas a camisinha e de 29,7\% que usam mais de um método contraceptivo. Com esses dados nota-se que os adolescentes precisam de educação sexual para evitar agravos futuros (Felisbino et al., 2018).

A adolescência é a fase que ocorre as mudanças hormonais, nas mulheres com o desenvolvimento corporal a primeira menstruação (menarca), nos homens a mudança no tom da voz, crescimento dos pelos, mudanças no psicológico em busca da identidade psicossocial, mudanças no comportamento e no pensamento (Mds,2013). A atividade sexual na adolescência tornase possível, e quando desprotegido estão expostas as Infecções Sexualmente Transmissíveis (IST) e/ou uma gravidez indesejada. Orientações e programas de educação sexual são importantes durante essa fase, fazendo planejamento e intervenções durante o acompanhamento aos adolescentes, tornando-os conscientes quanto aos fatores de risco (Rêgo et al., 2018).

De acordo com a Nova Escola, Paulo Freire é tido como o mentor da educação para a consciência. Freire foi um filosofo e educador brasileiro que acreditava no ensino de troca, onde o professor e alunos aprendiam com as informações, onde o aluno não é um receptor de conteúdo, o aprendizado é feito em conjunto, os dois lados aprende. É importante desenvolver a educação para saúde do adolescente, pois engloba o conhecimento dos adolescentes e a troca entre o educador e o educando (Ferrari, 2008).

Educação permanente é uma das atribuições do enfermeiro, faz-se necessário para a prestação de cuidado da população e integração na equipe multiprofissional, respeitando a individualidade e realidade da comunidade, a troca de conhecimento é fundamental nesse processo, visando melhorar a qualidade de vida e contribuindo para melhoria da equipe de saúde (Silva et al., 2015). 
O ministério da saúde possui a caderneta de saúde do adolescente, nela está estabelecida que a adolescência é dada entre 10 e 19 anos de idade, onde ocorre as mudanças hormonais, descobertas, autocuidado, transformações no corpo (Mds,2013). Já no estatuto da criança e do adolescente (ECA), a idade considerada é entre 12 e 18 anos de idade. Considerando que os serviços de saúde levam em conta que aos 10 anos de idade inicia as mudanças no corpo (ECA, 2019).

O ambiente escolar e familiar contribui para o desenvolvimento pessoal, durante a transição para adolescência é importante o apoio dos pais e do enfermeiro para planejamento de vida saudável e definição de estratégias para prevenir comportamento de riscos e promoção da saúde mental (Aparicio et al., 2020).

Entre todas as experiências vividas pelo adolescente, as primeiras relações sexuais são as mais importantes. A vida sexual geralmente tem início na adolescência e por isso eles estão mais vulneráveis as infecções sexualmente transmissíveis e uma gravidez não planejada. Sendo assim a importância do profissional de saúde na educação sexual dando orientações sobre o uso correto dos métodos contraceptivos e sobre as patologias ocasionadas pelo ato sexual (Borges et al., 2016).

O vírus da imunodeficiência humana é uma doença crônica que não tem cura e causa muitos agravos, transmitida através do uso incorreto ou não uso dos métodos contraceptivos. É uma das maiores preocupações das autoridades em saúde, já que essa condição traz mudanças na vida do adolescente diante da necessidade de tratamento do vírus HIV (Levandowski et al., 2016).

O início das relações sexuais para os adolescentes é interpretado como uma oportunidade de autonomia, que torna-os mais suscetível a Infecções Sexualmente Transmissíveis (IST) como por exemplo o aumento de casos entre jovens de 15 a 24 anos com HIV positivo, devido a múltiplos parceiros sexuais, uso incorreto ou não uso de contraceptivos, podendo ocasionar em uma gestação não planejada devido à falta de segurança na prática sexual (Maranhão et al., 2017).

O decreto $n^{\circ}$ 6.286, 5 de dezembro de 2007. Instituiu o Programa Saúde na Escola (PSE), onde o ministério da saúde juntamente com o ministério da educação trabalha juntos, trocando informações sobre a saúde dos estudantes. Profissionais de saúde vão até a escola de rede pública realizar visitas periódicas para proporcionar atendimento à saúde durante o ano letivo, por meio de ações de prevenção, promoção educação e atenção à saúde (Silva, 2007).

O ministério da saúde utiliza do projeto saúde e prevenção nas escolas, para realizar ações de promoção da saúde sexual e da saúde reprodutiva de adolescentes e jovens, contribuindo com a redução do número de pessoas infectadas por IST, e também pela evasão escolar devido à gravidez não planejada. Devido a falta do uso de preservativo as IST podem causar infertilidade, doença inflamatória pélvica, câncer de colo uterino, gravidez ectópica, interferindo na vida dos adolescentes (Krabbe et al., 2016).

O papel da enfermagem diante dos programas voltados para saúde do adolescente visa a promoção, prevenção de doenças e agravos, de forma biopsicossocial "se inicia com mudanças corporais na puberdade e termina com a integração social e a independência econômica desse indivíduo", levando em consideração as reais necessidades educacionais e assistenciais em saúde, como puberdade, sexualidade, gravidez (Silva et al., 2016).

O programa de saúde do adolescente (PROSAD) foi criado para prestar serviços direcionando a melhora de saúde dos adolescentes, através das práticas educativas, tratamento e reabilitação, em busca de melhorar o atendimento, diminuir os conflitos e esclarecer questionamentos, prestando assistência adequada a cada situação com a participação do adolescente no planejamento (Henriques et al., 2010).

Segundo Potter Perry a enfermagem é importante para o desenvolvimento dos adolescentes, ajudando através das orientações quanto a educação em saúde. "Por exemplo, a enfermeira conduz seminários em uma escola de ensino médio oferecendo sugestões práticas para solução de problemas do interesse de um grande grupo de alunos" (Perry, 2012).

A taxa de fertilidade entre os jovens é alta devido à falta de informação, aspectos culturais e sociais, baixa escolaridade e renda. A Pesquisa Nacional de Saúde do Escolar (PENSE) permite aproximações para conhecer melhor o perfil da saúde sexual e reprodutiva dos adolescentes. A PENSE é um inquérito conduzido a cada três ano, desde 2009, pelo Instituto Brasileiro de 
Geografia e Estatística (IBGE), em parceria com o Ministério da Saúde, com estudantes de escolas públicas e privadas com intuito de analisar os indicadores (Felisbino et al., 2018).

Acredita-se que a saúde sexual dos adolescentes está relacionada com o papel do estado, na falta de investimento em ações educativas, poucos recursos básicos, desigualdade social. A falta de orientação tornando a adolescente mais vulnerável a gestação, e a falta de instrução e apoio se tratando da paternidade ao adolescente do sexo masculino. Que são obrigados a ter responsabilidade e amadurecer nessa fase da vida (Schaefer et al., 2018).

A gravidez na adolescência é considerada um problema de saúde pública devido aos impactos causados pelas causados pelas consequências biológicas, psicológicas, econômicas, educacionais e familiares e de saúde. A adolescente grávida tem direito ao acompanhamento pré-natal com médicos e enfermeiros na Unidade básica de saúde, mas muitas por terem medo de represália mantém-se caladas até o final da gestação colocando o bebê e a si própria em risco (Queiroz et al., 2016).

A lei 13.257, de 2016 assegura "a todas as mulheres o acesso aos programas e as políticas de saúde da mulher, e de planejamento reprodutivo e, às gestantes, nutrição adequada, atenção humanizada à gravidez, ao parto e ao puerpério e atendimento pré-natal, perinatal e pós-natal integral no âmbito do Sistema Único de Saúde” (ECA, 2019).

A assistência pré-natal é importante para toda gestante, deve-se orientar quanto a nutrição, a ingesta de alimentos in natura, ricos em vitaminas $\mathrm{A}, \mathrm{E}, \mathrm{D}, \mathrm{C}$ e dos minerais cálcio, fósforo e magnésio, dessa forma prevenindo quanto a diabetes gestacional, pré-eclâmpsia, prematuridade, diminuindo o risco de o bebê nascer com baixo peso, promovendo saúde e reduzindo agravos (Belfort et al., 2018).

A infecção pelo HIV entre jovens é crescente, a facilidade em iniciar a vida sexual, o ambiente familiar, convívio com a violência são considerados situações de risco. A educação em saúde contribui para conhecimento, prevenção, promoção e recuperação em saúde, transformando o comportamento dos adolescentes através das ações, intervenções, discussões e palestras sobre o conhecimento dos métodos contraceptivos e uso dos preservativos com objetivo de diminuir os casos de ISTs (Monteiro et al., 2019).

As ações realizadas por profissionais através de projeto, palestras ajuda a compreender e aproximar os adolescentes quanto ao planejamento da prevenção e promoção de saúde, discutindo sobre higiene, gravidez, funcionamento do corpo feminino, as ISTs, e assim melhorar nos hábitos dos adolescentes, esclarecer as dúvidas, e conscientizar quanto a educação em saúde (Gonçalves et al., 2016).

O estudo com questionário relacionado a mulheres adolescentes grávidas e não grávidas de um município do nordeste do Brasil constatou que a cultura, comportamento, baixa escolaridade e baixa renda são indicadores de uma gestação na adolescência em mulheres menores que 19 anos (Pinheiro et al., 2019).

A vacina conta HPV tornou-se muito importante no Brasil, pois ela previne um dos cânceres mais letais, o do colo uterino. O câncer uterino está associado ao HPV, que é considerado uma infecção sexualmente transmissível (IST). Com isso a necessidade de intervenções educativas para promover informação adequada, mostrando aos adolescentes a importância da vacinação antes de começarem a vida sexual (Cruz et al., 2019).

Adolescentes em situação de rua tem uma maior probabilidade de gravidez e aborto, por iniciarem a vida sexual precoce em troca de dinheiro, favores ou vantagens. Quanto mais cedo o rompimento da ligação familiar e desvinculo escolar, maior a possibilidade de uma gravidez, devido a vulnerabilidade aos fatores de risco, o não uso de preservativo, e a falta de acesso aos métodos contraceptivos (Silva et al., 2018).

A gravidez não planejada na adolescência pode levar a decisões inseguras, o aborto, muitas vezes por pressão familiar, ou relato do companheiro para realizar esse procedimento. Não tendo apoio gestacional, a adolescente pode acabar acatando e tentar o abortamento, através de medicamentos: Misoprostol, Cytotec ou preparos caseiros: chás, folhas ou ervas (Maranhão et al., 2016). 
Durante o período de pré e pós-natal é importante o apoio às mães adolescentes (matriz de apoio), uma tríade psíquica: mãe/bebê/vó do bebê, com um papel principal e protetor, uma identificação experiente, próxima, e feminina de cuidado, facilitando o conhecimento materno a adolescente através das experiências da mãe/avó de maneira positiva (Frizzo et al., 2019).

A segunda gravidez na adolescência tem relação com a não conscientização dos métodos contraceptivos, falta de planejamento familiar e/ou a possibilidade de um aborto. Para que haja uma assistência positiva é necessário que a família ofereça apoio, participe da vida sexual orientando, conversando e ajudando nas decisões quanto aos projetos da vida (Inacio et al., 2016).

Adolescentes grávidas que passaram ou passam por situação de violência intrafamiliar necessita de um acolhimento, da família, do estado (através de terapia psicológica, pré-natal seguro) e/ou do companheiro, que com esse apoio ela consiga viver em um ambiente bom que se sinta protegida, e desenvolver o cuidado com o bebê tendo uma relação entre mãe e filho (Miura et al., 2018).

A sífilis é uma IST e quando associada à gravidez na adolescência torna-se um grave problema de saúde pública (materno-infantil), necessitando de cuidados especiais no pré-natal, a fim de prevenir a transmissão vertical (da mãe para o feto). A educação sexual realizada por profissionais de enfermagem é a principal medida de intervenção em combate as infecções sexualmente transmissíveis (Monteiro et al., 2015).

A matéria de saúde do adolescente durante o curso de graduação foi uma da maior motivação devido a abordagem sobre a mudança de comportamento, as mudanças corporais, as orientações sexuais que são feitas nessa fase, que aprimorou o conhecimento com a leitura de artigos, leis, caderneta do adolescente e estudos sobre o tema.

O presente estudo tem como objetivo verificar o papel do enfermeiro no cuidado a saúde do adolescente, descrevendo ações prestadas para auxiliá-los em casos de gestação precoce, e enfatizar a importância de utilizar métodos contraceptivos para prevenção de infecções sexualmente transmissíveis.

\section{Metodologia}

O presente estudo trata-se de uma revisão integrativa de literatura. A revisão integrativa é um tipo de metodologia que revisa dados da literatura afim de unir conhecimento, e propor uma análise do tema abordado, através das práticas baseadas em evidências (Souza, 2010). A prática baseada em evidências é um ponto importante na revisão integrativa, avalia os estudos e investiga através dos resultados, além de facilitar o entendimento do tema abordado ao leitor (Pompeo, 2009).

Para realização da revisão integrativa foi necessária seguir seis fases: elaboração da pergunta norteadora: sabe-se que na adolescência a reprodução sexual se torna possível, o que leva a um adolescente em idade fértil a ter uma gravidez precoce sem considerar os riscos de ISTs?, busca ou amostragem na literatura; coleta de dados; análise crítica dos estudos incluídos, discussão dos resultados, apresentação da revisão integrativa (Souza, 2010).

A pesquisa inicial para compor o presente trabalho buscou artigos relacionados ao tema, após as leituras, foram escritos resumos dos textos cujo tema estava relacionado ao papel do enfermeiro no cuidado a saúde do adolescente, que se utiliza das ações educativas para orientá-los quanto a promoção da saúde, as infecções sexualmente transmissíveis, a gravidez precoce, e sensibilizar o uso correto dos métodos contraceptivos.

Foram selecionados e analisados o total de 23 artigos, nas bases de dados SCIELO-Scientific Eletronic Library Online, LILACS-Literatura Latino Americana e do Caribe em Ciências da Saúde, BDENF-Base de Dados em Enfermagem, utilizando as palavras chaves disponíveis no Decs-Descritores em ciências da saúde: saúde do adolescente, educação em saúde, gravidez na adolescência, métodos contraceptivos, infecções sexualmente transmissíveis. 
Para a seleção dos artigos foram utilizados os seguintes critérios de inclusão: artigos que estavam associados a temática abordada, publicados em português e entre 2010-2020, que continham o texto disponível na íntegra. Como critérios de exclusão foram utilizados: artigos publicados antes de 2010, artigos estrangeiros, e artigos em que apenas o resumo estava disponível.

Após a análise do conteúdo selecionado buscou-se ordenar as informações encontradas de modo a evidenciar o papel da enfermagem nas ações educativas relacionadas a saúde do adolescente e educação sexual de forma que possibilitassem a encontrar a resposta ao problema estudado. Após os dados decorrentes das análises realizadas foram confrontados com o referencial teórico do estudo.

Não foi necessário passar pelo comitê de ética por se tratar de uma revisão integrativa de literatura, priorizando o conhecimento, analisando os resultados e evidências por meio do levantamento bibliográfico sobre a saúde dos adolescentes, sendo assim um instrumento válido para enfermagem.

\section{Resultados e Discussão}

Constatou-se que $100 \%$ dos artigos foram publicados em periódicos nacionais. Selecionados artigos que estavam associados a temática abordada, publicados em português e que continham o texto disponível na íntegra, representado por estudo de caso, relato de experiência, e observação dos indicadores.

Na classificação dos estudos feitos, os indicadores obtidos foram concentrados em 10 artigos, a iniciação sexual e uso de métodos contraceptivos (4 artigos), gravidez na adolescência (3 artigos), e a importância do profissional de saúde no acompanhamento dos casos (3 artigos). Foram divididos em 50\% em estudos quantitativos e $50 \%$ qualitativos.

Em relação aos anos de publicação dos artigos, compreendidos entre 2010 e 2019, os dados coletados apresentaram a distribuição, a seguir, apresentado no Quadro 1. 
Research, Society and Development, v. 10, n. 14, e503101422498, 2021

(CC BY 4.0) | ISSN 2525-3409 | DOI: http://dx.doi.org/10.33448/rsd-v10i14.22498

Quadro 1. Dados coletados em artigos selecionados para o estudo.

\begin{tabular}{|c|c|}
\hline AUTOR, ANO DE PUBLICAÇÃO, PAÍS & $\begin{array}{c}\text { BREVE DESCRIÇÃO SOBRE A METODOLOGIA E } \\
\text { SOBRE A PESQUISA }\end{array}$ \\
\hline Belfort et al. (2018), Brasil & $\begin{array}{l}\text { Analise que visou identificar os determinantes do baixo peso } \\
\text { em filhos de adolescentes ao nascer. }\end{array}$ \\
\hline Borges et al. (2016), Brasil & $\begin{array}{l}\text { Estudo de caso que estimou prevalências de iniciação sexual } \\
\text { e uso de métodos contraceptivos na última relação sexual de } \\
\text { adolescentes brasileiros. }\end{array}$ \\
\hline Cruz et al. (2019), Brasil & $\begin{array}{c}\text { Pesquisa descritiva com abordagem qualitativa que analisou } \\
\text { a percepção dos adolescentes sobre o HPV. }\end{array}$ \\
\hline Felisbino-Mendes et al. (2018), Brasil & $\begin{array}{c}\text { Analisou os indicadores de saúde sexual e reprodutiva de } \\
\text { adolescentes com base nos dados da Pesquisa Nacional de } \\
\text { Saúde do Escolar. }\end{array}$ \\
\hline Henriques et al. (2010), Brasil & $\begin{array}{c}\text { Estudo por meio de metodologia qualitativa que objetivou } \\
\text { conhecer o significado que os profissionais da atenção } \\
\text { primária dão ao atendimento do adolescente. }\end{array}$ \\
\hline Krabbe et al. (2016), Brasil & $\begin{array}{l}\text { Estudo que relatou a experiência da residência } \\
\text { multiprofissional em Saúde da Família e Comunidade } \\
\text { através da execução de atividade de educação em saúde com } \\
\text { adolescentes sobre o tema Infecção Sexualmente } \\
\text { Transmissível (IST) e sexualidade. }\end{array}$ \\
\hline Maranhão et al. (2017), Brasil & $\begin{array}{l}\text { Analisou a repercussão da iniciação sexual de jovens com } \\
\text { antecedentes obstétricos no número de gestações e de } \\
\text { parceiros. }\end{array}$ \\
\hline Monteiro et al. (2015), Brasil & $\begin{array}{l}\text { Estudo observacional que teve objetivo de investigar fatores } \\
\text { associados à sífilis entre adolescentes. }\end{array}$ \\
\hline Neiva-Silva et al. (2018), Brasil & $\begin{array}{l}\text { Estudo que identificou a prevalência de experiência de } \\
\text { gravidez e aborto e os fatores associados em adolescentes na } \\
\text { situação de rua. }\end{array}$ \\
\hline Queiroz et al. (2017), Brasil & $\begin{array}{l}\text { Estudo qualitativo, descritivo que descreveu o cuidado de } \\
\text { enfermagem no pré-natal após a implementação do grupo de } \\
\text { gestantes adolescentes norteado pelas expectativas e } \\
\text { experiências de adolescentes grávidas. }\end{array}$ \\
\hline
\end{tabular}

Fonte: Autores (2021).

Avaliando estudo nacional de Borges et al. (2016) com 74.589 adolescentes estudantes, com ênfase sobre a iniciação sexual e uso de métodos contraceptivos na última relação sexual (preservativo masculino e pílula anticonceptiva oral). Observouse que $28,1 \%$ dos adolescentes tinham iniciado a vida sexual, com maior prevalência naqueles de17 anos. Entre adolescentes que tinham iniciado a vida sexual, $82,3 \%$ referiram uso de métodos contraceptivos na última relação sexual. O preservativo masculino foi usado por $68,8 \%$ sem diferença por tipo de escola. Pílula anticoncepcional foi utilizada por $13,4 \%$, sendo mais frequente entre mulheres de 17 anos. Com isso nota-se que os adolescentes têm conhecimento da prevenção sexual, a maioria faz uso de métodos contraceptivos nas relações, assim diminuindo os riscos de infecções sexualmente transmissíveis com o uso do preservativo masculino.

Analisando estudos feitos por Monteiro et al. (2015), entre os anos de 2003 e 2012 onde foram realizados 33665 atendimentos, sendo que 10,40\% desse público eram adolescentes de ambos os sexos, 1183 do sexo feminino e 664 do sexo masculino. Comparando adolescentes gestantes com aquelas não gestantes, as respostas mostraram-se diversas proporções entre os grupos. A presença de IST (gestantes: 5,5\%, não gestantes: 26,7\%), uso de drogas (gestantes: 8,9\% e não gestantes: 26,6\%), multiplicidade de parceiros sexuais (gestantes: 14,4\% e não gestantes: 37,8\%). A prevalência de sífilis, na adolescência, foi de 
Research, Society and Development, v. 10, n. 14, e503101422498, 2021

(CC BY 4.0) | ISSN 2525-3409 | DOI: http://dx.doi.org/10.33448/rsd-v10i14.22498

0,86\% (30), com proporções de 1,95\% (13) para o sexo masculino, 1,18\% (14) para o feminino e 0,18\% (3) para gestantes. Notase que a relação sexual com múltiplos parceiros e o não uso do preservativo é o principal fator de exposição à sífilis, independente do status sorológico, a relação sexual e a baixa prevalência do uso do preservativo são as principais formas de exposição à sífilis em ambos os sexos e gestantes adolescentes.

No ano de 2015 Felisbino et al. (2018) realizou um estudo comparativo para analisar os indicadores de saúde sexual e reprodutiva dos adolescentes do nono ano escolar. Notou-se que a iniciação sexual teve queda de 30,5\% (2009) para 27,5\% (2015), 75,9\% para 66,2\% quanto ao uso do preservativo. Além da diminuição na orientação para prevenção de gravidez nas escolas públicas de $81,1 \%$ para $79,3 \%$. Cerca de $30 \%$ relataram uso combinado de preservativo e outro método, e 19,5\% não fizeram uso de método algum. Apesar da diminuição no início da vida sexual, os adolescentes estão mais vulneráveis as ISTs e a gravidez precoce devido ao baixo índice na porcentagem da prevenção e a diminuição de orientação sexual.

Uma pesquisa realizada por Krabbe et al. (2016), com 441 adolescentes de ambos os sexos com participação ativa dos mesmos através de aplicação de questionário. Notou-se que dentre os jovens entrevistados $74 \%$ dizem já terem tido relações sexuais. $73 \%$ dos jovens relatam terem usado preservativo na primeira relação sexual e $27 \%$ diz não terem utilizado preservativo. Foi questionado a eles quais IST podem ser transmitidas por via sexual, 87\% acreditam que o HIV é transmitido por via sexual, já em relação ao HPV 67\% acham que é transmitido por relação sexual, quanto á sífilis 65\% é transmitida por relação sexual e á herpes 52\% acreditam que é transmitido por via sexual. Ao serem questionados em relação ao preservativo $93 \%$ diz que o preservativo é um método contraceptivo para evitar gravidez e 67\% acreditam que a pílula é um método contraceptivo. Pode-se observar que os adolescentes têm iniciado as suas relações sexuais mais precocemente, a falta de conhecimento pode levar a práticas que comprometam a saúde do jovem com isso contribui para o aumento do número de casos de alguma IST. Por isso é de total relevância adotar medidas de educação sexual e tratar diferentes assuntos nos setores de saúde que trabalham com esses adolescentes, para que não aja nenhum agravo em sua qualidade de vida, e proporcionar assim educação sexual.

De acordo com o estudo de Belfort et al. (2018) com 751 adolescentes gestantes atendidas na maternidade do Rio de Janeiro, resultou que a não aceitação da gravidez, ter menos de seis consultas de pré-natal, não ter assistência nutricional padronizada, e o parto prematuro são possíveis determinantes para o bebê nascer com baixo peso. Nota-se que a gravidez na adolescência precisa de um apoio devido a não aceitação, é necessário que a unidade básica de saúde faça acolhimento e busca ativa das gestantes que faltaram nas consultas marcadas, afim de prevenir e reduzir os riscos.

Em análise feita por Maranhão et al. (2017) com adolescentes que iniciaram a relação sexual precoce constatou-se que $50,6 \%$ das adolescentes pesquisadas afirmaram ter menstruado entre os 12 e 13 anos e uma em cada cinco tinham engravidado pelo menos três vezes $(19,4 \%$ ) e 49,1\% era primigesta A primeira relação sexual afirmou que ocorreu entre 9 e 19 anos. Quando falado do número de parceiros sexuais, $42,2 \%$ das entrevistadas relataram que tiveram um único parceiro sexual. A orientação das adolescentes sobre gravidez e IST foi dada na escola $(72,2 \%)$ e mais da metade das adolescentes entrevistadas informaram não terem procurado algum tipo de orientação no serviço de saúde antes de ter a primeira relação sexual (78\%). Com isso alguns fatores podem ter tido alguma interferência na idade da primeira relação sexual como: a escolaridade, a idade da menarca, o tempo de namoro com o primeiro parceiro sexual e a orientação sexual da escola. A antecipação da iniciação sexual tem sido apontada como fator relevante para uma série de implicações negativas na vida dos adolescentes como exposição á IST e gravidez não planejada. Com isso observou-se que os adolescentes dessa pesquisa não fizeram uso do método anticoncepcional na sua primeira relação sexual, pois às decisões sobre as práticas contraceptivas dos adolescentes que são tomadas no início da vida sexual implicara no seu futuro e qualidade de vida.

Em uma entrevista realizada por Neiva-Silva et al. (2018) com 307 adolescentes e jovens em situação de rua, sendo a maioria dos adolescentes do sexo masculino (81,1\%). 239 relatam já terem tido relações sexuais e começaram entre os 12 e 14 anos. A experiência de gravidez (ter ficado grávida ou ter engravidado alguém) ocorreu em aproximadamente um terço da 
amostra (29,3\%). 42,2\% já tiveram experiência de gravidez, e 26,7\% tiveram mais de uma vez. Apenas 57,8\% fez algum exame pré-natal durante a gravidez. $\mathrm{Na}$ análise foram identificados alguns fatores de risco para a gravidez, como o aborto, ter duas ou mais gravidezes. Com isso fica evidente a necessidade que essa população descrita necessita para ter uma educação sexual eficaz para diminuir o número de gravidez não planejada.

Em um estudo de Henriques et al. (2010) participou 12 profissionais de saúde (5 médicos e 7 enfermeiros que atuavam nas equipes e atendiam o adolescente na sua rotina de trabalho há mais de um ano no município de Viçosa, Minas Gerais). Conclui-se que:

1- $\quad$ O atendimento realizado pelos profissionais, contribui para a melhoria da atenção prestada aos adolescentes em nível primário.

2- Os profissionais de saúde relataram por meio dos discursos, que atender ao adolescente é desafiante.

3- É necessário que a atenção seja ampliada e realizada de forma integral.

4- $\quad \mathrm{O}$ atendimento aos adolescentes, poderá propiciar novos conhecimentos que contribuirão de forma significativa para a elaboração de futuras ações que envolvam os profissionais de saúde e os adolescentes.

Assim fica evidenciado a importância dos profissionais de saúde participarem de ações educativas que tenham troca de informações, e melhore o conhecimento dos adolescentes.

Queiroz et al. (2016) realizou estudo em unidade de atenção primária de Fortaleza, com 16 adolescentes gestantes, tinham idade entre 14 e 19 anos, (dez adolescentes eram primigestas, três secundigestas, duas tercigestas e uma quadrigesta). Todas com número de consultas de pré-natal adequado para a idade gestacional. Foi relatado por adolescentes que a maneira como o enfermeiro mediou os grupos, teve impacto positivo para elas, além de estimular momentos de reflexões das experiências já vivenciadas e das realidades a serem enfrentadas. Onde aquelas que já eram mães compartilharam as suas experiências com as demais, que puderam compreender melhor o fenômeno. O enfermeiro ter feito atividades de interação e diálogo entre as adolescentes foi ótimo, identificou as necessidades e apoiou as jovens para cuidar do filho e também olhar para si. Dessa forma o compartilhamento de saberes e troca de experiência gerou o interesse entre as participantes e garantiu o dinamismo grupal facilitando o aprendizado.

Foi realizado uma entrevista por Cruz et al. (2019) para avaliar o conhecimento dos adolescentes entre 12 e 14 anos a respeito do HPV, atendidos numa unidade básica de saúde do Amapá. Foi dividido grupos por meio de categorias, onde: a primeira abordou o conhecimento dos adolescentes a respeito da dor da vacina e quais as patologias ela previne, a segunda sobre o saber dos adolescentes quanto o câncer de colo uterino e a terceira sobre o conhecimento dos benefícios da vacina. O resultado foi uma falta de informação pelos adolescentes comprometendo assim na adesão à vacina. Diante disso, nota-se a importância de um suporte de informações de acordo com o nível de desenvolvimento deles, ou seja, faz-se necessária uma abordagem de socialização sobre as informações no nível de compreensão dos adolescentes, sendo assim os adolescentes precisam receber as informações de forma adequada à sua capacidade de entendimento para conseguir transformá-las em práticas que os protejam.

\section{Considerações Finais}

Conclui-se que o início sexual entre os adolescentes está cada vez mais precoce, apesar do conhecimento sobre os métodos contraceptivos e da prevenção sexual uma parte desses jovens não faz uso, assim se expondo a uma provável infecção sexualmente transmissível ou uma gravidez não planejada. Para diminuir os casos de ISTs é necessário que os profissionais de saúde estejam engajados em ações que conscientize os adolescentes sobre a importância de se prevenir, e quanto aos agravos de saúde caso uma exposição a infecção. 
Research, Society and Development, v. 10, n. 14, e503101422498, 2021

(CC BY 4.0) | ISSN 2525-3409 | DOI: http://dx.doi.org/10.33448/rsd-v10i14.22498

A gravidez precoce necessita de apoio e assistência para mãe e o bebê, nota-se que a maioria dos casos acontece devido à baixa escolaridade, relação sexual com múltiplos parceiros, e a falta de orientação assim tornando irrelevante o uso dos métodos para prevenção. Com isso, podemos enfatizar que a gestação na adolescência precisa de uma dedicação maior, para conscientizar quanto a importância do pré-natal, a aceitação da gravidez, e a prevenção das ISTs, tornando-os mais responsáveis.

A participação efetiva dos profissionais de saúde, principalmente do enfermeiro, nas ações de conscientização aos adolescentes é de extrema importância, para desenvolver conhecimento, orientá-los com informações corretas sobre os métodos contraceptivos e infecções sexualmente transmissíveis, atendendo de forma ampliada, mas respeitando a necessidade de cada adolescente.

O enfermeiro deve fazer consultas, palestras, grupos para troca de informações e experiências com adolescentes, afim de avaliar as necessidades e compartilhar o conhecimento. Entender a realidade dos jovens é primordial para realizar a orientação em saúde e manter um diálogo sobre sexualidade.

Nota-se que esse estudo contribui para enfermagem, evidenciando o papel do enfermeiro na educação sexual dos adolescentes, no desenvolvimento de técnicas para orientação e promoção de saúde, na troca de informações sobre gestação não planejada, no uso correto dos métodos contraceptivos, e nos riscos de infecções sexualmente transmissíveis, utilizando a necessidade dos adolescentes como base das discussões.

Após essas afirmações, é necessário levar adiante os estudos e pesquisas, sobre o papel do enfermeiro na educação sexual dos adolescentes, fazendo reformulação e atualização dessa temática.

\section{Referências}

Aparício, G. (2020). Estudo comparativo da percepção de resiliência por pais e crianças/adolescentes. Acta Paulista de Enfermagem, São Paulo. 33(20190178), $1-8$.

Belfort, G. P. (2018). Determinantes do baixo peso ao nascer em filhos de adolescentes. Ciência \& Saúde Coletiva , Rio de Janeiro. 23(8), 2609-2620.

Brasil. (2019). Estatuto da Criança e do Adolescente: Lei n ${ }^{\circ} 8.069$, de 13 de julho de 1990. Conanda. 5-230.

Borges, A. L. V. (2019) O início da vida sexual e contracepção em adolescentes brasileiros. Revista de Saúde Pública, São Paulo. 50(1), 1-11.

Cruz, M. N. M (2019). Vacina hpv: Percepção de adolescentes atendidos em uma unidade básica de saúde do amapá. Enfermagem em Foco. Macapá. 10(2), $136-141$.

Felisbino, M. M. S. (2018). Análise dos indicadores de saúde sexual: e reprodutiva de adolescentes brasileiros, 2009 , 2012 e 2015 . Revista Brasileira de Epidemiologia, São Paulo. 21(1),1-14.

Frizzo, G. B. (2019). Maternidade Adolescente: A Matriz de Apoio e o Contexto de Depressão Pós-Parto. Psicologia: Teoria e Pesquisa, Brasília. 35(3533), 113.

Gonçalves, L. F. F. (2016). Promoção de saúde com adolescentes em ambiente escolar: relato de experiência. Sanare, Ceará. 15(2).

Henriques, B. D., Rocha, R. L., \& Madeira, A. M. F. (2010). Saúde do adolescente: o significado do atendimento aos profissionais da atenção primária do município de Viçosa, MG. Revista Médica de Minas Gerais, Minas Gerais. 20(3), 300-309.

Inácio, A. L. R., \& Rasera, E. F. (2016). Repetição da "gravidez na adolescência" e do planejamento familiar. Psicologia, Saúde \& Doenças, Lisboa. 17(2), 179188.

Krabbe, E. C. (2016). Escola, Sexualidade, Práticas Sexuais e Vulnerabilidade: Para as Infecções Sexualmente Transmissíveis (IST). Revista Interdisciplinar de Ensino Pesquisa e Extensão.4(1).

Levandowski, D. C. (2016). Adolescência e HIV/AIDS: Uma análise bibliométrica da produção científica brasileira no período 1980-2013. Revista Adolescência e Saúde, Rio de Janeiro. 13(1).

Maranhão, T. A. (2017). Repercussão da iniciação sexual na vida sexual e reprodução de jovens de capital do Nordeste brasileiro. Ciência \& Saúde Coletiva, Rio de Janeiro. 22(12), 4083-4094.

Maranhão, T. A., Gomes, K. R. O., \& Barros, I. D. C. (2016). Fatores preditores do abortamento entre jovens com experiência obstétrica. Revista Brasileira de Epidemiologia, São Paulo. 19(3).

Miura, P. O., Tardivo, L. S. D. L. P. C., \& Barrientos, D. M. S. (2018). O desamparo vivenciado por mães adolescentes e adolescentes grávidas acolhidas institucionalmente. Ciência saúde coletiva, Rio de Janeiro. 23(5), 1601-1610. 
Research, Society and Development, v. 10, n. 14, e503101422498, 2021

(CC BY 4.0) | ISSN 2525-3409 | DOI: http://dx.doi.org/10.33448/rsd-v10i14.22498

Monteiro, M. D. O. P. (2015). Fatores associados à ocorrência de sífilis em: adolescentes do sexo masculino, feminino e gestantes de um Centro de Referência Municipal/CRM - DST/HIV/AIDS de Feira de Santana, Bahia. Revista Adolescência e Saúde, Rio de Janeiro.12(3), 21-32.

Monteiro, R. S. D. M. (2019). Ações educativas sobre prevenção de HIV/AIDS entre adolescentes em escolas. Enfermería Atual da Costa Rica, San José. 3(37), 206-222,

Neiva, S. L. (2018). Experiência de gravidez e aborto em crianças, adolescentes e jovens em situação de rua. Ciência \& Saúde Coletiva, Rio de Janeiro. 23(4), 1055-1066.

Ferrari, M. (2008). Paulo Freire, o mentor da Educação para a consciência. Nova Escola.

Perry, P. A. P. A. G. (2012). Fundamentos de Enfermagem. Rio de Janeiro. 3, 1-1445.

Pinheiro, Y. T., Pereira, N. H., \& Freitas, G. D. D. M. (2019). Fatores associados à gravidez em adolescentes de um município do nordeste do Brasil. Cadernos saúde coletiva, Rio de Janeiro, 27(4), 363-367.

Pompeo, D. A., Rossi, L. A., \& Galvão, C. M. (2009). Revisão integrativa: etapa inicial do processo de validação de diagnóstico de enfermagem. Acta Paulista de Enfermagem, São Paulo, 22(4), 434-438.

Brasil. Decreto $n^{\circ}$ 6.286, de 5 de dezembro de 2007 - Institui o Programa Saúde na Escola - PSE, e dá outras providências.

Queiroz, M. V. O. (2027). Grupo de gestantes adolescentes: contribuições para o cuidado no pré-natal. Revista Gaúcha de Enfermagem. 37(2016), 1-7.

Rêgo, M. H., Cavalcanti, A., \& Maia, E. (2018). Resiliência e gravidez na adolescência: uma revisão integrativa. Psicologia, Saúde \& Doenças, Lisboa. 19(3), 710-723.

Saúde, M. D. (2013). Caderneta de Saúde da Adolescente: Ministério da Saúde. (2a . ed.) Brasília: Biblioteca Virtual em Saúde do Ministério da Saúde. 4-50.

Silva, J. K. O., Anjos, D. F., Pimentel, P. S., Costa, I. M. G., \& Fonseca, J. H. M. Identidade de gênero e orientação sexual: sexualidade no contexto escolar. Pesquisa, Sociedade e Desenvolvimento , [S. l.]. 8(8), e12881182

Schaefer, R. (2018). Políticas de Saúde de adolescentes e jovens no contexto luso-brasileiro: especificidades e aproximações. Abrasco, Rio de Janeiro. 23(9), 2849-2857.

Silva, C. S. O. E. (2016). O adolescente na Estratégia Saúde da Família: uma revisão integrativa de literatura. Adolescência e Saúde, Rio de Janeiro. 13(3), 7687.

Silva, R. C. D. (2015). O papel do enfermeiro como educador e pesquisador, e a integração entre prática baseada em evidências e educação permanente. Revista interdisciplinar da PUC Minas no Barreiro, Belo Horizonte. 5(10), 417-430.

Souza, M. T. D., Silva, M. D. D., \& Carvalho, R. (2010). Revisão integrativa: o que é e como fazer. Einstein, São Paulo. 8(1), 102-106. 